\title{
Análise Comparativa de Parâmetros Hidráulicos para Dimensionamento de Tecnologias em Estações de Tratamento de Esgoto
}

\author{
Daniella de Lima Moraes'; Ana Silvia Pereira Santos'; Daniele Maia Bilal; Luis Carlos Soares da \\ Silva Junior ${ }^{2}$ Bruna Magalhães Araujo ${ }^{l}$ \\ $\bowtie$ daniellalmoraes@gmail.com
}

1. Universidade do Estado do Rio de Janeiro - UERJ. 2. Universidade Federal do Rio de Janeiro - UFRJ.

Histórico do Artigo:

Recebido em: 14 de outubro de 2019

Aceito em: 20 de janeiro de 2020

Publicado em: 30 de abril de 2020

Resumo: As tecnologias de tratamento de esgotos, combinadas entre si, devem propiciar o melhor desempenho de uma Estação de Tratamento de Esgoto (ETE), dentro de suas condições operacionais. Para o dimensionamento hidráulico e sanitário de cada tecnologia, são utilizados diferentes parâmetros e um maior entendimento desses, contribui para implantação de ETEs mais eficientes. Neste sentido o presente trabalho realizou uma análise comparativa entre os parâmetros hidráulico sanitário por meio de rearranjos das equações de dimensionamento do decantador primário, decantador secundário, reator UASB e seu compartimento de decantação, além das lagoas anaeróbia, facultativa e de polimento. Para tanto foram abordados os seguintes parâmetros: Taxa de Aplicação Superficial (TAS), Tempo de Detenção Hidráulica (TDH), Taxa de Aplicação Orgânica (TA0), Carga Orgânica Volumétrica $(\mathrm{COV})$ e Profundidade $(\mathrm{H})$. Por fim foram simuladas diversas condições experimentais, por meio da variação das faixas limite de valores propostos nas diversas bibliografias adotadas. Os resultados do estudo mostraram que algumas condições estabelecidas a partir de valores recomendados na NBR 12.209:2011 e na literatura não são aplicáveis e/ou viáveis de serem implementadas. Em termos de tratamento anaeróbio, o reator UASB se mostrou o mais eficiente, como previsto. Assim como, os resultados encontrados para as lagoas facultativas e de polimento, que confirmam o princípio de tratamento adotado em cada unidade. Quanto aos resultados encontrados a respeito dos decantadores primários e reatores UASB, através dos cálculos teóricos, foi possível observar que existem condições que permitem a adoção de áreas mais reduzidas, quando elevadas as profundidades, mantendo a eficiência do sistema de tratamento.

Palavras-chave: Taxa de aplicação superficial, Carga orgânica volumétrica, Taxa de aplicação orgânica, Tempo de detenção hidráulica, Profundidade.

\section{Comparative Analysis of Hydraulic Design Parameters in Sewage Treatment Plants Technologies}

\begin{abstract}
The combination of wastewater treatment process should provide the best performance of a Waste Water Treatment Plant (WWTP) within its operational conditions. For the hydraulic and sanitary design of each technology, different parameters are often used and a deeper understanding of these contributes to the implementation of more efficient STPS. Hence, the present work performed a comparative analysis between the sanitary hydraulic parameters by rearranging the design equations for: primary sedimentation tank, secondary clarifier, UASB reactor and its decantation compartment, besides the anaerobic, optional and polishing lagoons. The following parameters were addressed: Hydraulic Load (HL), Hydraulic Retention Time (HRT), Specific Surface Area (SSA), Applied B0D Flux (ABF) and Depth (D). Subsequently, several experimental conditions were simulated by varying the limit values proposed in the various adopted bibliographies. The study results revealed that some conditions established from recommended values are neither applicable nor feasible to be implemented. In terms of anaerobic treatment, the UASB reactor proved the most efficient as predicted. Furthermore, the results found for facultative and polishing lagoons confirmed the treatment principle adopted in each unit. Regarding the results found related to primary sedimentation tanks and UASB reactors, through the theoretical calculations, it was possible to observe that there are conditions allowing the adoption of smaller areas, when the depths are higher, maintaining the efficiency of the treatment system.
\end{abstract}

Keywords: Hydraulic load, Hydraulic retention time, Specific surface area, Applied bod flux and depth. 


\section{Análisis Comparativo de Parámetros Hidráulicos para Tecnologías de Dimensión en Estaciones de Tratamiento de Aguas Residuales}

Resumen: La utilizacion de varios procesos de tratamiento de aguas residuales combinados entre sí, deben proporcionar el mejor rendimiento de una planta de tratamiento de aguas residuales (PTAR). Para el dimensionamiento hidráulico y sanitario de cada tecnología, se utilizan diferentes parámetros y una mayor comprensión de estos contribuye a la implementación de PTAR más eficientes. El presente trabajo realizó un análisis comparativo entre los parámetros hidráulicos sanitarios mediante la reordenación de las ecuaciones de dimensionamiento del decantador primario, decantador secundario, reactor UASB y su compartimento de decantación, además de las lagunas anaerobias, facultativas y de pulido. Se abordaron los siguientes parámetros: tasa de aplicación superficial (TAS), tiempo de detención hidráulica (TDH), tasa de aplicación orgánica (TA0), carga orgánica volumétrica $(\mathrm{COV})$ y profundidad $(\mathrm{P})$. Se simularon varias condiciones de experimental variando los valores límite propuestos en las diversas bibliografías adoptadas. Los resultados del estudio mostraron que algunas condiciones establecidas a partir de valores recomendados en NBR 12.209: 2011 y en literatura no son aplicables o factibles de implementar. En términos de tratamiento anaeróbico, el reactor UASB demostró ser el más eficiente según lo previsto. Además de los resultados encontrados para lagunas facultativas y de pulido, que confirman el principio de tratamiento adoptado en cada unidad. Con respecto a los resultados encontrados sobre los decantadores primarios y los reactores UASB, a través de los cálculos teóricos, fue posible observar que existen condiciones que permiten la adopción de áreas más pequeñas, cuando las profundidades son más altas, manteniendo la eficiencia del sistema de tratamiento.

Palabras clave: Tasa de aplicación superficial, Carga orgánica volumétrica, Tasa de aplicación orgánica, Tiempo de mantenimiento hidráulico, Profundidad.

\section{INTRODUÇÃo}

No Brasil, no ano de 2017, apenas $60,2 \%$ da população urbana era atendida com rede coletora de esgoto. Dessa porcentagem, somente 73,7\% eram encaminhados para algum tipo de tratamento antes da disposição final em corpos receptores, ou seja, aproximadamente $44,0 \%$ do total de esgotos gerados (BRASIL, 2019). Dados apresentados pela Agência Nacional de Águas (ANA) indicam que 43,0\% da população possui seus esgotos coletados e tratados em Estações de Tratamento de Esgotos (ETEs), além de 12,0\% da população ser atendida por solução individual. Dessa forma, 55,0\% da população possui atendimento adequado (ANA, 2017).

0 tratamento de esgoto permite o emprego de diversos processos e tecnologias, que podem ser combinados entre si, com o objetivo de atender as restrições legais, assegurar a saúde pública e o bem-estar da população, além de garantir a proteção ambiental. A qualidade da operação e manutenção, combinada com a concepção adequada tem impacto crucial nos resultados do sistema de esgotamento sanitário. Além de atender os objetivos técnicos, o sistema deve conciliar baixos custos de implementação e operação (HELLER; CASTR0, 2013; CHERNICHAR0, 2016).

Nesse sentido, o emprego de maiores taxas de aplicação de matéria orgânica e aplicação hidráulica propiciam reduções no tempo de detenção hidráulica em reatores de tratamento de esgotos. Essa redução impacta diretamente na redução de volumes dos mesmos, resultando na 
economia de implantação da tecnologia (KUCZMAN, 2011). Essa opção propicia ETEs mais compactas de forma a ocupar menores áreas. Isso influencia diretamente no custo de aquisição de terreno e no custo de material para construção levando à investimentos mais reduzidos. Entretanto, unidades com maior introdução de tecnologia podem levar a custos operacionais mais elevados em função de maiores índices de mecanização.

De forma a quantificar os possíveis cenários de redução nas áreas ocupadas pelas estações, se faz necessário o entendimento dos fatores que envolvem a modelagem dos processos de tratamento de esgotos e correlacioná-los. Para tal, serão avaliados os principais parâmetros para dimensionamento hidráulico-sanitário de unidades físico-biológicas do tratamento de esgotos consolidadas no Brasil e estabelecidos na literatura (Jordão e Pessoa, 2017; Von Sperling, 2014; Von Sperling, 2017; Chernicharo, 2016) e na NBR 12.209:2011, que dispõe sobre as condições de elaboração de projetos hidráulico-sanitário de estações de tratamento de esgotos. Os principais parâmetros de dimensionamento hidráulico-sanitário utilizados para dimensionamento de unidades são: Taxa de Aplicação Superficial (TAS), Taxa de Aplicação Orgânica (TA0), Carga Orgânica Volumétrica (COV), Profundidade (H) e Tempo de Detenção Hidráulica (TDH).

Desse modo, o presente trabalho visa analisar comparativamente esses parâmetros através do rearranjo das equações clássicas de dimensionamento das diferentes tecnologias de tratamento. Para avaliar a correlação entre os parâmetros foram simuladas diversas condições experimentais teóricas, por meio da variação dentro da faixa de valores propostos nas diversas bibliografias já citadas e na NBR 12.209:2011.

\section{MATERIAL E MÉTODOS}

0 trabalho foi desenvolvido com base em interações entre equações básicas de dimensionamento hidráulico-sanitário de tecnologias de tratamento de esgotos. As tecnologias adotadas foram: decantadores primário e secundário (do tipo circular), reatores anaeróbios de fluxo ascendente em manta de lodo - Upflow Anaerobic Sludge Blanket (UASB), compartimento de decantação do reator UASB, lagoa anaeróbia, lagoa facultativa e lagoa de polimento. Inicialmente foram adotadas as Equações de 01 a 05 de dimensionamento hidráulico-sanitário, citadas por Jordão e Pessoa (2017). Posteriormente, foram realizadas associações entre os parâmetros de dimensionamento, de maneira segregada entre os processos compactos e os processos naturais, com a finalidade de avaliar as relações entre eles. 
$T A S=Q / A$

(Equação 01)

$C=Q \times C_{o}$

$\operatorname{COV}=C / \mathrm{V}$

(Equação 03)

TAO $=C / A$

(Equação 04)

$T D H=V / Q$

(Equação 05)

Onde:

TAS - Taxa de Aplicação Superficial $\left(\mathrm{m}^{3} \cdot \mathrm{m}^{-2} \cdot \mathrm{d}^{-1}\right)$;

Q - Vazão $\left(\mathrm{m}^{3} \cdot \mathrm{d}^{-1}\right)$; A - Área ( $\mathrm{m}^{2}$ ou ha);

C - Carga Orgânica (kg DB0.d $\left.\mathrm{d}^{-1}\right)$;

$\mathrm{C}_{0}$ - Concentração de DB0 (mg.. $\left.{ }^{-1}\right)$;

COV - Carga Orgânica Volumétrica $\left(\mathrm{kg}\right.$ DBO $\left.0 \mathrm{~m}^{-3} \cdot \mathrm{d}^{-1}\right)$;

$\mathrm{V}$ - Volume $\left(\mathrm{m}^{3}\right)$;

TA0 - Taxa de Aplicação Orgânica (kg DBO.ha- $\left.{ }^{-1} \cdot \mathrm{d}^{-1}\right)$;

TDH - Tempo de Detenção Hidráulica (h ou d).

Observação: As lagoas de estabilização aqui avaliadas são processos naturais, que ocupam grandes áreas e demandam elevados tempos de detenção hidráulica, em relação aos demais processos compactos. Dessa forma, para estes casos, os parâmetros “área” e "TDH” são abordados em termos de hectares (ha) e dias (d), respectivamente. De maneira oposta, para os sistemas compactos, os parâmetros "área" e "TDH" são abordados em termos de metros quadrados (m2) e horas (h), respectivamente. Destaca-se que as características dos sistemas compactos não foram comparadas às das lagoas de estabilização, simplesmente por não serem comparáveis entre si.

Dessa forma, as Equações 01 a 05 foram combinadas e foram adotados os valores estabelecidos na NBR 12.209:2011 e na literatura (VON SPERLING, 2017; VON SPERLING, 2014; JORDÃO E PESSOA, 2017; CHERNICHAR0, 2016). Essa literatura foi adotada em função de apresentar valores já consolidados para os parâmetros definidos. Cabe ressaltar que alguns valores extremos foram adotados somente para avaliar as condições limites das tecnologias.

Como as unidades de tratamento apresentam métodos de dimensionamento distintos e valores de parâmetros pré-estabelecidos, optou-se, neste trabalho, por realizar as combinações dos parâmetros em função das unidades de tratamento.

Na Tabela 1, podem ser observados os parâmetros de dimensionamento hidráulicosanitário recomendados para as diferentes unidades de estudo. 
Tabela 1. Parâmetros de dimensionamento hidráulico-sanitário recomendados para as diferentes unidades

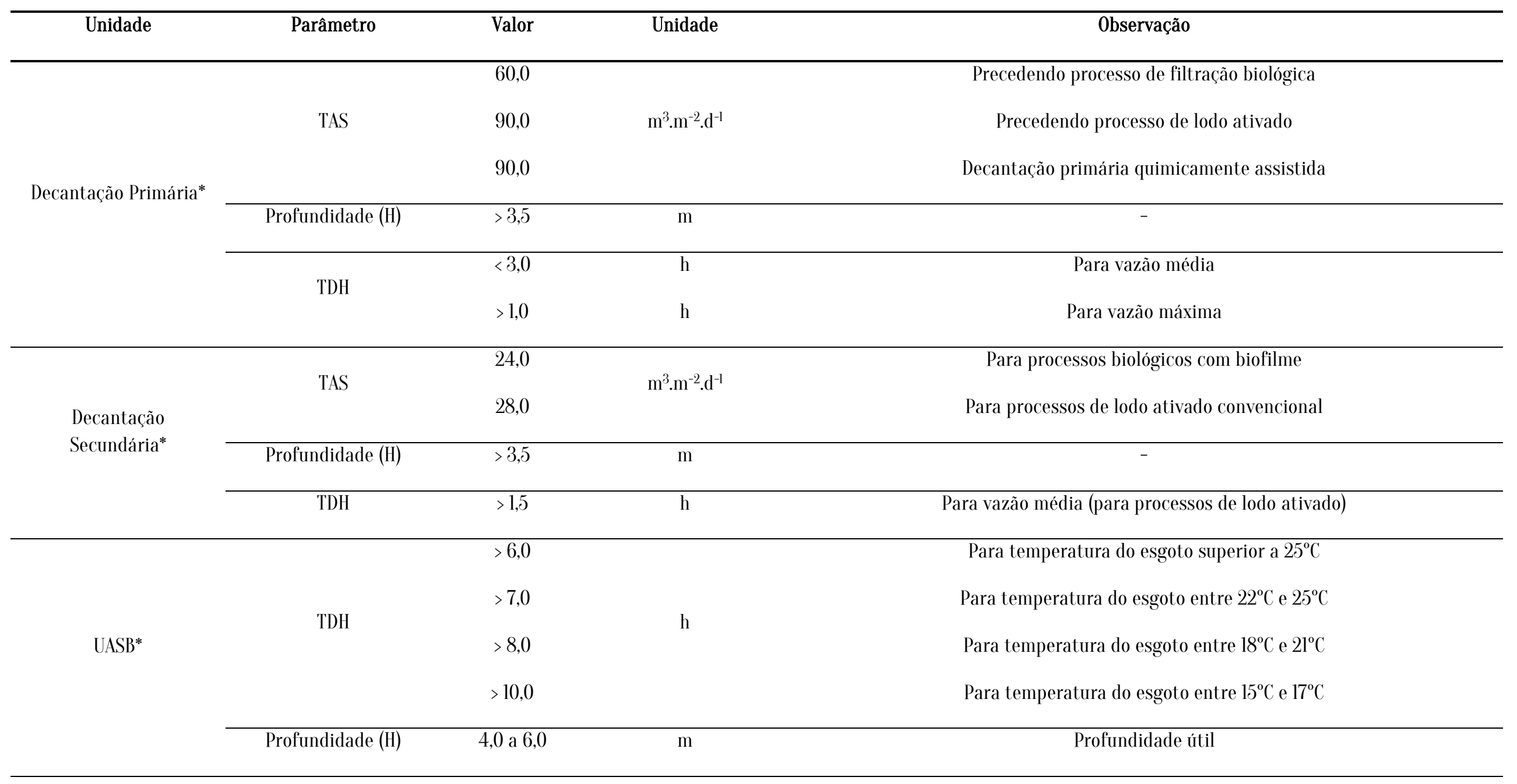

Revista Internacional de Ciências, v. 10, n. 01, p. 22-41, jan-abr, 2020 


\begin{tabular}{|c|c|c|c|c|}
\hline \multirow{3}{*}{$\begin{array}{l}\text { Compartimento de } \\
\text { decantação dos UASB }\end{array}$} & TAS & $<1,2$ & $\mathrm{~m}^{3} \cdot \mathrm{m}^{-2} \cdot \mathrm{h}^{-1}$ & $\begin{array}{c}\text { Para vazão máxima } \\
\text { (Equivalente a } 28,0 \mathrm{~m}^{3} \cdot \mathrm{m}^{-2} \cdot \mathrm{d}^{-1} \text { ) }\end{array}$ \\
\hline & Profundidade $(\mathrm{H})$ & $>1,5$ & $\mathrm{~m}$ & Profundidade útil \\
\hline & TDH & $>1,0$ & $\mathrm{~h}$ & Para vazão máxima \\
\hline \multirow{12}{*}{$\begin{array}{l}\text { Lagoas anaeróbias, } \\
\text { facultativas e de } \\
\text { polimento }{ }^{* *}\end{array}$} & \multirow{3}{*}{$\mathrm{COV}$} & $0,10-0,30$ & $\mathrm{kgDB} 0 \cdot \mathrm{m}^{-3} \cdot \mathrm{d}^{-1}$ & Para lagoa anaeróbia (Temperatura entre $10^{\circ} \mathrm{C}$ e $20^{\circ} \mathrm{C}$ ) \\
\hline & & $0,30-0,35$ & $\mathrm{kgDB} 0 . \mathrm{m}^{-3} \cdot \mathrm{d}^{-1}$ & Para lagoa anaeróbia (Temperatura entre $20^{\circ} \mathrm{C}$ e $25^{\circ} \mathrm{C}$ ) \\
\hline & & 0,35 & $\operatorname{kgDB} 0 \cdot \mathrm{m}^{-3} \cdot \mathrm{d}^{-1}$ & Para lagoa anaeróbia (Temperatura acima de $25^{\circ} \mathrm{C}$ ) \\
\hline & \multirow{3}{*}{ TA0 } & $100-180$ & $\operatorname{kgDBO} 0 \cdot \mathrm{ha}^{-1} \cdot \mathrm{d}^{-1}$ & Lagoa Facultativa - Inverno frio e baixa insolação \\
\hline & & $120-240$ & $\operatorname{kgDBO} 0 \cdot \mathrm{ha}^{-1} \cdot \mathrm{d}^{-1}$ & Lagoa facultativa - Inverno e insolação moderados \\
\hline & & $240-350$ & $\mathrm{kgDB} 0 \cdot \mathrm{ha}^{-1} \cdot \mathrm{d}^{-1}$ & Lagoa facultativa - Inverno Quente e elevada insolação \\
\hline & \multirow{2}{*}{ Profundidade (H) } & $3,0-5,0$ & $\mathrm{~m}$ & Lagoa anaeróbia \\
\hline & & $1,5-2,0$ & $\mathrm{~m}$ & Lagoa facultativa \\
\hline & \multirow{4}{*}{ TDH } & $0,5-1,0$ & d & Lagoa de polimento \\
\hline & & $3-6$ & d & Lagoa anaeróbia \\
\hline & & $15-45$ & d & Lagoa facultativa \\
\hline & & $9-12$ & d & Lagoa de polimento \\
\hline
\end{tabular}

Fonte: Adaptado de NBR 12.209:2011 (ABNT, 2011); Von Sperling (2017); Chernicharo (2016).

Revista Internacional de Ciências, v. 10, n. 01, p. 22-41, jan-abr, 2020 


\section{Decantador primário}

Para os decantadores primários (DP), a NBR 12.209:2011 traz recomendações de valores para os parâmetros TAS, profundidade (H) e TDH (Tabela 1). Todavia, não são propostos valores de referência para a aplicação da COV nessa unidade de tratamento, por se tratar de um processo físico de sedimentação e não de decomposição de matéria orgânica.

Foram realizadas interações entre as Equações 01, 02 e 03, gerando uma nova equação, denominada Equação 06.

$\operatorname{COV}=\frac{(T A S \times C o)}{H}$

(Equação 06)

Onde: $\mathrm{H}$ - profundidade (m).

A partir daí, foram estabelecidas para simulação, as condições experimentais apresentadas na Tabela 2.

Tabela 2. Condições experimentais utilizadas para cálculo do COV em DP

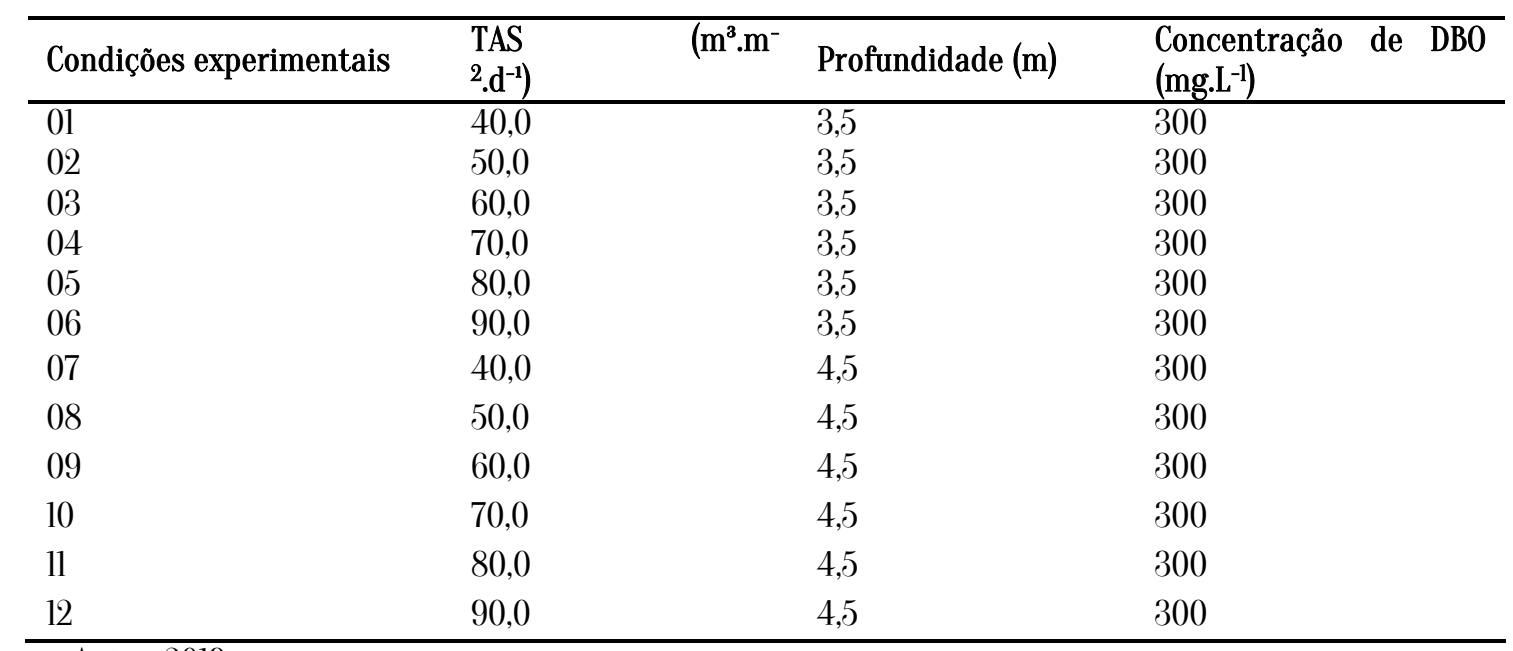

Fonte: Autor, 2019.

\section{Decantador secundário}

Para os decantadores secundários (DS), a NBR 12.209:2011 apresenta recomendações de valores para os parâmetros TAS, profundidade e TDH, em função da unidade precedente no fluxograma de tratamento (Tabela 1). De maneira análoga ao decantador primário, não são propostos valores de referência para a aplicação da carga orgânica volumétrica. Neste caso, 
Análise Comparativa de Parâmetros Hidráulicos para Dimensionamento de Tecnologias em Estações de Tratamento de Esgoto

apesar de o objetivo dessa unidade ser de remoção se sólidos orgânicos, o processo também é físico e se dá por sedimentação. Assim, nessa unidade também não há preocupação com 0 processo de decomposição de matéria orgânica e sim com a sua remoção por sedimentação.

Para análise do decantador secundário, também foi adotada a Equação 06. Assim, a partir desta, foram estabelecidas para simulação, as condições experimentais apresentadas na Tabela 3.

Tabela 3. Condições experimentais utilizadas para cálculo do COV em DS

\begin{tabular}{lllll}
\hline Condições experimentais & TAS & $\left(\mathrm{m}^{3} \cdot \mathrm{m}^{-2} \cdot \mathrm{d}^{-1}\right)$ & Profundidade $(\mathrm{m})$ & $\begin{array}{l}\text { Concentração de } \\
\left(\mathrm{mg} . \mathrm{L}^{-1}\right)\end{array}$ \\
\hline 01 & 20,0 & & 3,5 & 195 \\
02 & 24,0 & 3,5 & 195 \\
03 & 28,0 & 3,5 & 195 \\
04 & 20,0 & 4,5 & 195 \\
05 & 24,0 & 4,5 & 195 \\
06 & 28,0 & 4,5 & 195 \\
\hline
\end{tabular}

Fonte: Autor, 2019 .

\section{Reator UASB}

Para os reatores UASB, a NBR 12.209:2011 recomenda valores para os parâmetros TDH e profundidade (Tabela 1). No entanto, não são propostos valores de referência para carga orgânica volumétrica e para taxa de aplicação superficial. Ressalta-se que no caso dessa tecnologia, a NBR 12.209:2011 ainda sugere outros parâmetros de verificação, como velocidade ascensional e tempo de detenção hidráulica no compartimento de digestão. Entretanto, por não se adequarem ao escopo desse trabalho, estes não serão analisados.

Neste caso, inicialmente foram associadas as Equações 01 e 05, formando a Equação 07. Ressalta-se que na Equação 05, o volume (V) foi substituído pelo produto entre área (A) e profundidade (H). Posteriormente foram realizadas interações entre as Equações 02, 03 e 05, gerando uma nova equação, denominada Equação 08.

$$
\begin{aligned}
& T A S=\frac{H}{T D H} \\
& C O V=\frac{C_{O}}{T D H}
\end{aligned}
$$

A partir das Equações 07 e 08, foram estabelecidas para simulação, as condições experimentais apresentadas na Tabela 4. 
Tabela 4. Condições experimentais utilizadas para cálculo do COV e TAS em reatores UASB

\begin{tabular}{llll}
\hline Condições experimentais & TDH $(\mathrm{h})$ & Profundidade $(\mathrm{m})$ & $\begin{array}{l}\text { Concentração de DB0 } \\
\text { (mg.L.' }\end{array}$ \\
\hline 01 & 6,0 & 4,0 & 300 \\
02 & 7,0 & 4,0 & 300 \\
03 & 8,0 & 4,0 & 300 \\
04 & 9,0 & 4,0 & 300 \\
05 & 10,0 & 4,0 & 300 \\
06 & 6,0 & 6,0 & 300 \\
07 & 7,0 & 6,0 & 300 \\
08 & 8,0 & 6,0 & 300 \\
09 & 9,0 & 6,0 & 300 \\
10 & 10,0 & 6,0 & 300 \\
\hline
\end{tabular}

Fonte: Autor, 2019.

\section{Compartimento de decantação do reator UASB}

De modo a promover uma discussão sobre os diferentes processos físicos de sedimentação, foi adotado também o compartimento de decantação dos reatores UASB. Para este, a NBR 12.209:2011 apresenta recomendações de valores para os parâmetros TAS, profundidade e TDH, (Tabela 1). De maneira análoga aos decantadores primário e secundário, para este processo não são propostos valores de referência para a aplicação da COV, pois esta é uma etapa física do processo completo que ocorre no reator UASB.

Para o compartimento de decantação do Reator UASB, foi adotada a Equação 06. Assim, foram estabelecidas para simulação, as condições experimentais apresentadas na Tabela 5.

Tabela 5. Condições experimentais utilizadas para cálculo do COV em compartimentos de decantação de reatores UASB

\begin{tabular}{lllll}
\hline Condições experimentais & TAS & $\left(\mathrm{m}^{3} \cdot \mathrm{m}^{-2} \cdot \mathrm{d}^{-1}\right)$ & Profundidade $(\mathrm{m})$ & $\begin{array}{l}\text { Concentração de DB0 } \\
\left(\mathrm{mg} . \mathrm{L}^{-1}\right)\end{array}$ \\
\hline 01 & 20,0 & 1,5 & 300 \\
02 & 24,0 & 1,5 & 300 \\
03 & 28,0 & 1,5 & 300 \\
04 & 20,0 & 3,5 & 300 \\
05 & 24,0 & 3,5 & 300 \\
06 & 28,0 & 3,5 & 300 \\
07 & 20,0 & 5,0 & 300 \\
08 & 24,0 & 5,0 & 300 \\
09 & 28,0 & 5,0 & 300 \\
\hline
\end{tabular}

Fonte: Autor, 2019. 


\section{Lagoas de estabilização}

As lagoas de estabilização, apesar de representarem o maior número de processos de tratamento por ETEs no Brasil (ANA, 2017), não são regulamentadas pela NBR 12.209:2011. Por isso, os parâmetros empregados em seus projetos de dimensionamento são estabelecidos somente na literatura. Ressalta-se que no presente trabalho serão analisadas as lagoas anaeróbias (LAn), facultativa (LF) e de polimento (LP).

Von Sperling (2017) aborda princípios para o dimensionamento das lagoas anaeróbias, trazendo recomendações de valores para os parâmetros COV, TDH e profundidade. Contudo, não são apresentados valores de referência para o parâmetro TAS. Parâmetros de dimensionamento semelhantes aos das lagoas anaeróbias são empregados para as lagoas facultativas. Von Sperling (2017) apresenta recomendações de valores também para os parâmetros TDH e profundidade. Porém, em relação à taxa de aplicação orgânica, no caso das lagoas facultativas, se dá em função da área da superfície líquida e não do volume. Assim, o valor recomendado por Von Sperling (2017) é para TA0 e não para COV. E, o único parâmetro que não têm valores de referência definidos na literatura, de maneira análoga à lagoa anaeróbia, também é a TAS. Para o caso das lagoas de polimento, Von Sperling (2017) recomenda somente valores de TDH e profundidade. Não são propostos valores de referência para TAS, e aplicação orgânica, tanto em função da área superficial líquida como em função do volume.

Assim, para os três tipos de lagoas de estabilização estuadas no presente trabalho, foram realizadas três interações entre as equações básicas. Na primeira, a interação foi entre as Equações 01, 02 e 03, gerando a Equação 09. Na Equação 03, o volume (V) foi substituído pelo produto entre área (A) e profundidade (H). A Equação 10 foi gerada a partir das Equações 01 e 05 e é similar à Equação 07, porém com a ordem dos fatores alterada. Por fím, a Equação 11 foi gerada a partir de uma nova interação entre as Equações 01, 02 e 04.

$$
\begin{aligned}
& T A S=\frac{(\operatorname{COV} x H)}{C_{O}} \\
& T D H=\frac{H}{T A S} \\
& T A S=\frac{T A O}{C_{O}}
\end{aligned}
$$


Para as lagoas anaeróbias, a partir das Equações 09 e 10, foram estabelecidas para simulação, as condições experimentais da Tabela 6 .

Tabela 6. Condições experimentais utilizadas para cálculo de TAS em Lan

\begin{tabular}{|c|c|c|c|}
\hline Condições experimentais & $\operatorname{COV}\left(\mathrm{KgDB} 0 . \mathrm{m}^{3} \cdot \mathrm{d}^{-1}\right)$ & Profundidade (m) & $\begin{array}{l}\begin{array}{l}\text { Concentração de DB0 } \\
\left(\mathrm{mg} . \mathrm{L}^{-1}\right)\end{array} \\
\end{array}$ \\
\hline 01 & 0,10 & 3,0 & 300 \\
\hline 02 & 0,10 & 4,0 & 300 \\
\hline 03 & 0,10 & 5,0 & 300 \\
\hline 04 & 0,20 & 3,0 & 300 \\
\hline 05 & 0,20 & 4,0 & 300 \\
\hline 06 & 0,20 & 5,0 & 300 \\
\hline 07 & 0,30 & 3,0 & 300 \\
\hline 08 & 0,30 & 4,0 & 300 \\
\hline 09 & 0,30 & 5,0 & 300 \\
\hline 10 & 0,35 & 3,0 & 300 \\
\hline 11 & 0,35 & 4,0 & 300 \\
\hline 12 & 0,35 & 5,0 & 300 \\
\hline
\end{tabular}

Fonte: Autor, 2019.

Para as lagoas facultativas, a partir das Equações 09 e 11, foram estabelecidas para simulação, as condições experimentais da Tabela 7.

Tabela 7. Condições experimentais utilizadas para cálculo de TAS em LF

\begin{tabular}{llll}
\hline Condições experimentais & TA0 $\left(\mathrm{KgDB0} \mathrm{ha}^{-1} \cdot \mathrm{d}^{-1}\right)$ & Profundidade $(\mathrm{m})$ & $\begin{array}{l}\text { Concentração de DB0 } \\
\left(\mathbf{m g . L} \mathrm{L}^{-1}\right)\end{array}$ \\
\hline 01 & 100,0 & 1,5 & 300 \\
02 & 120,0 & 1,5 & 300 \\
03 & 180,0 & 1,5 & 300 \\
04 & 240,0 & 1,5 & 300 \\
05 & 350,0 & 1,5 & 300 \\
06 & 100,0 & 2,0 & 300 \\
07 & 120,0 & 2,0 & 300 \\
08 & 180,0 & 2,0 & 300 \\
09 & 240,0 & 2,0 & 300 \\
10 & 350,0 & 2,0 & 300 \\
\hline
\end{tabular}

Fonte: Autor, 2019.

Para as lagoas de polimento, a partir das Equações 07 e 08, foram estabelecidas para simulação, as condições experimentais da Tabela 8. Para este caso, diferentes dos demais, adotou-se concentração média de DB0 de $90 \mathrm{mg} . \mathrm{L}^{-1}$, considerando que a lagoa de polimento é utilizada após um processo de reator UASB, onde o efluente já sofreu uma remoção de carga orgânica de aproximadamente 70\%, conforme descrito por Von Sperling (2014). 
Análise Comparativa de Parâmetros Hidráulicos para Dimensionamento de Tecnologias em Estações de Tratamento de Esgoto

Tabela 8. Condições experimentais utilizadas para cálculo de COV e TAS em LP

\begin{tabular}{llll}
\hline Condições experimentais & TDH (d) & Profundidade $(m)$ & $\begin{array}{l}\text { Concentração de DB0 } \\
\left(\text { mg. } L^{-1}\right)\end{array}$ \\
\hline 01 & 9,0 & 0,5 & 90 \\
02 & 12,0 & 0,5 & 90 \\
03 & 9,0 & 1,0 & 90 \\
04 & 12,0 & 1,0 & 90 \\
\hline
\end{tabular}

Fonte: Autor, 2019.

\section{RESULTADOS E DISCUSSÃ0}

A Tabela 9 compila os resultados dos cálculos realizados para determinação dos parâmetros definidos nas condições experimentais adotadas para as tecnologias de decantação primária e secundária. Cabe ressaltar que se encontram em destaque (negrito) os parâmetros que tem seus valores recomendados na NBR 12.209:2011 ou na literatura (VON SPERLING, 2017; VON SPERLING, 2014; CHERNICHAR0, 2016; JORDÃ0 E PESSOA, 2017). Além disso, apresentam-se tachados os parâmetros que não se encontram com os valores dentro dos limites propostos na NBR 12.209:2011 ou na literatura (VON SPERLING, 2017; JORDÃO E PESSOA, 2017).

Tabela 9. Resultados das condições experimentais adotadas para as tecnologias de decantação primária e secundária

\begin{tabular}{llllll}
\hline Tecnologia & Cond. & TAS & Prof. & TDH & COV \\
\hline & 01 & 40,0 & 3,5 & 2,1 & 3,43 \\
& 02 & 50,0 & 3,5 & 1,7 & 4,29 \\
& 03 & $\mathbf{6 0 , 0}$ & 3,5 & 1,4 & 5,14 \\
& 04 & $\mathbf{7 0 , 0}$ & 3,5 & 1,2 & 6,00 \\
Decantador & 05 & $\mathbf{8 0 , 0}$ & 3,5 & 1,1 & 6,86 \\
Primário & 06 & $\mathbf{9 0 , 0}$ & 3,5 & 0,9 & 7,71 \\
& 07 & $\mathbf{4 0 , 0}$ & $\mathbf{4 , 5}$ & 2,7 & 2,67 \\
& 08 & $\mathbf{5 0 , 0}$ & $\mathbf{4 , 5}$ & 2,2 & 3,33 \\
& 09 & $\mathbf{6 0 , 0}$ & $\mathbf{4 , 5}$ & 1,8 & 4,00 \\
& 10 & $\mathbf{7 0 , 0}$ & 4,5 & 1,5 & 4,67 \\
& 11 & $\mathbf{8 0 , 0}$ & $\mathbf{4 , 5}$ & 1,4 & 5,33 \\
& 12 & $\mathbf{9 0 , 0}$ & $\mathbf{4 , 5}$ & 1,2 & 6,00 \\
\hline \multirow{5}{*}{ Decantador } & 01 & $\mathbf{2 0 , 0}$ & 3,5 & 6,5 & 1,11 \\
Secundário & 02 & $\mathbf{2 4 , 0}$ & 3,5 & 5,4 & 1,34 \\
& 03 & 28,0 & 3,5 & 3,0 & 1,56 \\
& 04 & 20,0 & 4,5 & 8,3 & 0,87 \\
& 05 & 24,0 & 4,5 & 6,9 & 1,04 \\
& 06 & $\mathbf{2 8 , 0}$ & $\mathbf{4} 5$ & 3,9 & 1,20 \\
\hline
\end{tabular}

Observação: TAS $\left(\mathrm{m}^{3} \cdot \mathrm{m}^{-2} \cdot \mathrm{d}^{-1}\right) ; \mathrm{P}(\mathrm{m}) ; \mathrm{TDH}(\mathrm{d}) ; \operatorname{COV}\left(\mathrm{kgDBO} \cdot \mathrm{m}^{-3} \cdot \mathrm{d}^{-1}\right)$

Fonte: Autor, 2019. 
A Tabela 10 compila os resultados dos cálculos realizados para determinação dos parâmetros definidos nas condições experimentais adotadas para o reator UASB e seu compartimento de decantação.

Tabela 10. Resultados das condições experimentais adotadas para o reator UASB e seu compartimento de decantação

\begin{tabular}{|c|c|c|c|c|c|}
\hline Tecnologia & Cond. & TAS & Prof. & TDH & $\mathrm{COV}$ \\
\hline \multirow{9}{*}{$\begin{array}{l}\text { Comp. } \\
\text { decantação } \\
\text { reator UASB }\end{array}$} & 01 & 20,0 & 1,5 & 1,8 & 4,00 \\
\hline & 02 & 24,0 & 1,5 & 1,5 & 4,80 \\
\hline & 03 & 28,0 & 1,5 & 1,3 & 5,60 \\
\hline & 04 & 20,0 & 3,5 & 4,2 & 1,71 \\
\hline & 05 & 24,0 & 3,5 & 3,5 & 2,06 \\
\hline & 06 & 28,0 & 3,5 & 3,0 & 2,40 \\
\hline & 07 & 20,0 & 5,0 & 6,0 & 1,20 \\
\hline & 08 & 24,0 & 5,0 & 5,0 & 1,40 \\
\hline & 09 & 28,0 & 5,0 & 4,3 & 1,68 \\
\hline \multirow{10}{*}{ Reator UASB } & 01 & 16,00 & 4,0 & 6,0 & 1,20 \\
\hline & 02 & 13,71 & 4,0 & 7,0 & 1,03 \\
\hline & 03 & 12,00 & 4,0 & 8,0 & 0,90 \\
\hline & 04 & 10,67 & 4,0 & 9,0 & 0,80 \\
\hline & 05 & 9,60 & 4,0 & 10,0 & 0,72 \\
\hline & 06 & 24,00 & 6,0 & 6,0 & 1,20 \\
\hline & 07 & 20,57 & 6,0 & 7,0 & 1,03 \\
\hline & 08 & 18,00 & 6,0 & 8,0 & 0,90 \\
\hline & 09 & 16,00 & 6,0 & 9,0 & 0,80 \\
\hline & 10 & 14,40 & 6,0 & 10,0 & 0,72 \\
\hline
\end{tabular}

Observação: TAS $\left(\mathrm{m}^{3} \cdot \mathrm{m}^{-2} \cdot \mathrm{d}^{-1}\right) ; \mathrm{P}(\mathrm{m}) ; \mathrm{TDH}(\mathrm{d}) ; \mathrm{COV}\left(\mathrm{kgDB0} \cdot \mathrm{m}^{-3} \cdot \mathrm{d}^{-1}\right)$

Fonte: Autor, 2019.

A Tabela 11 compila os resultados dos cálculos realizados para determinação dos parâmetros definidos nas condições experimentais adotadas para as lagoas de estabilização.

Tabela 11. Resultados das condições experimentais adotadas para as lagoas de estabilização

\begin{tabular}{lllllll}
\hline Tecnologia & Cond. & TAS & Prof. & TDH & COV & TA0 \\
\hline \multirow{6}{*}{} & 01 & 1,00 & 3,0 & 72,0 & 0,10 & - \\
& 02 & 1,33 & 4,0 & 72,0 & 0,10 & - \\
& 03 & 1,67 & 5,0 & 72,0 & 0,10 & - \\
Lagoa & 04 & 2,00 & 3,0 & 36,0 & 0,20 & - \\
anaeróbia & 05 & 2,67 & 4,0 & 36,0 & 0,20 & - \\
& 06 & 3,33 & 5,0 & 36,0 & 0,20 & - \\
& 07 & 3,00 & 3,0 & 24,0 & 0,30 & - \\
& 08 & 4,00 & 4,0 & 24,0 & 0,30 & - \\
& 09 & 5,00 & 5,0 & 24,0 & 0,30 & - \\
& 10 & 3,5 & 3,0 & 20,6 & 0,35 & - \\
& 11 & 4,67 & 4,0 & 20,6 & 0,35 & - \\
& 12 & 5,83 & 5,0 & 20,6 & 0,35 & -
\end{tabular}


Estações de Tratamento de Esgoto

\begin{tabular}{lllllll}
\hline & 01 & 0,03 & 1,5 & 1080,0 & - & 100,0 \\
& 02 & 0,04 & 1,5 & 900,0 & - & 120,0 \\
& 03 & 0,06 & 1,5 & 600,0 & - & 180,0 \\
Lagoa & 04 & 0,08 & 1,5 & 450,0 & - & 240,0 \\
facultativa & 05 & 0,12 & 1,5 & 308,6 & - & 350,0 \\
& 06 & 0,03 & 2,0 & 1440,0 & - & 100,0 \\
& 07 & 0,04 & 2,0 & 1220,0 & - & 120,0 \\
& 08 & 0,06 & 2,0 & 800,0 & - & 240,0 \\
& 09 & 0,08 & 2,0 & 600,0 & - & 350,0 \\
\hline \multirow{3}{*}{ Lagoa } & 10 & 0,12 & 2,0 & 411,4 & - & - \\
polimento & 01 & 0,06 & 0,5 & 216,0 & 0,01 & - \\
& 02 & 0,04 & 0,5 & 288,0 & 0,01 & - \\
\hline
\end{tabular}

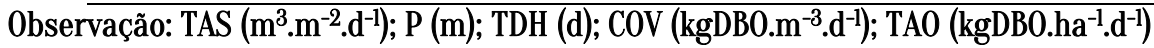

Fonte: Autor, 2019.

Segundo Santos et al. (2004), a sedimentação é uma das mais antigas técnicas aplicadas para a separação sólido-líquido e seu desempenho se baseia no tamanho e na massa específica das partículas que devem ser removidas. Entretanto, o principal parâmetro de dimensionamento das unidades de sedimentação (decantadores primários e secundários) é a taxa de aplicação superficial. Assim, apesar de a NBR 12.209:2011 indicar valores para TAS para o dimensionamento dessas unidades, alguns autores recomendam ensaios de coluna de sedimentação ou outra metodologia com o objetivo de melhorar a rotina de projetos de decantadores (SANTOS et al. 2004; BECKER et al., 1996). Substâncias especificas, como fármacos de uso geral, não são removidos em decantadores convencionais, já que as taxas comumente adotadas não são adequadas para partículas com essas características (G0BEL et al., 2007)

A eficiência da unidade de decantação primária está relacionada às condições de sedimentação e de retenção de sólidos. A condição de sedimentação depende da taxa de aplicação superficial. A retenção é controlada pelo tempo de detenção hidráulica, onde tempos de contato muito longos podem conduzir a uma condição de septicidade do esgoto, prejudicial para os processos subsequentes (FIGUEIRED0, 2009). Assim, para os decantadores primários, quanto menor a TAS, maior é o tempo que as partículas permanecem na unidade, permitindo que se obtenha uma maior eficiência de sedimentação. Essas condições só são observadas quando comparadas unidades que apresentam uma mesma profundidade útil para sedimentação das partículas.

Ainda em relação aos decantadores primários, quando comparadas as condições experimentais $04\left(\mathrm{TAS}=70 \mathrm{~m}^{3} \cdot \mathrm{m}^{-2} \cdot \mathrm{d}^{-1}\right.$ e $\left.\mathrm{H}=3,5\right)$ e $12\left(\mathrm{TAS}=90 \mathrm{~m}^{3} \cdot \mathrm{m}^{-2} \cdot \mathrm{d}^{-1}\right.$ e $\left.\mathrm{H}=4,5\right)$, é possível observar que essas alcançam o mesmo TDH e a mesma COV aplicada, assim apresentando, teoricamente, a mesma eficiência para o sistema. A partir desses resultados, é possível observar a afirmação realizada por Von Sperling (2017), onde o aumento da profundidade permite a 
adoção de uma unidade de decantação com menor área superficial (maior TAS), considerando uma mesma vazão aplicada, e mesma eficiência de sedimentação (mesmo TDH e COV aplicado).

Além disso, percebe-se que a NBR 12.209:2011 permite a adoção de valores superiores de taxa de aplicação superficial para os decantadores primários, em comparação aos aplicados para os decantadores secundários e compartimentos de decantação de reatores UASB. Isso, explicase pelos princípios de sedimentação adotados nesses sistemas, que são diferentes e estão atrelados às características das partículas sedimentáveis (CHAVEZ et al., 2006). Segundo Metcalf \& Eddy (2014), a sedimentação floculenta (característica dos decantadores primários) varia de acordo com a taxa de aplicação superficial e profundidade da unidade, como observado nos resultados, e pela concentração e faixas de dimensões das partículas. Deduz-se que quanto maior o número de partículas introduzidas no sistema, maior é o potencial que elas têm de se aglomerar, e assim maior a velocidade de sedimentação e consequentemente eficiência da unidade.

Percebe-se que os valores de TAS para os decantadores secundários e compartimentos de decantação dos reatores UASB apresentam valores muito similares, sendo que para os decantadores secundários é permitida a aplicação de uma taxa máxima de $24 \mathrm{~m}^{3} \cdot \mathrm{m}^{-2} \cdot \mathrm{d}^{-1}$ (para processos de filtração biológica) e $28 \mathrm{~m}^{3} \cdot \mathrm{m}^{-2} \cdot \mathrm{d}^{-1}$ (para processos de lodo ativado). Os compartimentos de decantação dos reatores UASB admitem uma taxa máxima de $28 \mathrm{~m}^{3} \cdot \mathrm{m}^{-2} \cdot \mathrm{d}^{-1}$.

Segundo Von Sperling (2016), a sedimentação dos decantadores secundários e consequente remoção de sólidos biológicos seguem o princípio da sedimentação zonal, onde se tem uma elevada concentração de sólidos, formando-se uma massa única que se sedimenta. Em relação ao processo de sedimentação do compartimento de decantação do reator UASB, este depende do tipo de lodo presente e das cargas aplicadas. Segundo Chernicharo (2016), o lodo pode ser do tipo floculento assumindo taxas de escoamento superficial inferiores ou do tipo granular podendo assumir taxas de escoamento superficial mais elevadas. Assim, para dimensionamento do compartimento de decantação do reator UASB, assume-se que a sedimentação é mais próxima da zonal, já que a taxa de escoamento superficial se assemelha àquela indicada para o decantador secundário. 0 compartimento de decantação do reator UASB, ainda tem uma particularidade em relação ao possível acúmulo de escuma na superfície líquida que pode prejudicar sobremaneira o desempenho do reator.

Segundo Almeida et al. (2018), uma forma de minimizar esse impacto, no projeto e na construção desse compartimento é garantir o nivelamento dos vertedores instalados nas canaletas de saída. 
Em tratamento de esgoto sanitário entende-se que uma parte significativa dos sólidos suspensos serão compostos de carga orgânica (VON SPERLING, 2014). Porém, nesse caso não se pode considerar que a remoção dessa carga orgânica entre 25 a 40\% expressa em DB0, conforme descrito por Metcalf \& Eddy (2014), será determinada em função das faixas de COV calculadas. Isso porque a remoção de matéria orgânica, introduzida nesse sistema, deve levar em conta que a DB0 global é a soma da DB0 dissolvida e a DB0 particulada e que o princípio do decantador primário é a remoção física das partículas suspensas. Ou seja, o percentual de remoção de DB0 dissolvida é aproximadamente o de redução dos sólidos suspensos totais, e por isso se a maior parte da DB0 total for solúvel, a eficiência de remoção de matéria orgânica será comprometida (FIGUEIRED0, 2009).

Encerrando a discussão sobre decantadores, observa-se que para a mesma TAS, ao se elevar a profundidade, eleva-se consequentemente o TDH. Assim, para se alcançar os valores de TDH máximos determinados pela NBR 12.209:2011 seria necessária a adoção de profundidades demasiadamente elevadas.

No caso dos reatores UASB, os resultados encontrados para as TAS variaram entre 9,6 $\mathrm{m}^{3} \cdot \mathrm{m}^{-2} \cdot \mathrm{d}^{-1}\left(0,4 \mathrm{~m}^{3} \cdot \mathrm{m}^{-2} \cdot \mathrm{h}^{-1}\right)$ e $24 \mathrm{~m}^{3} \cdot \mathrm{m}^{-2} \cdot \mathrm{d}^{-1}\left(1,0 \mathrm{~m}^{3} \cdot \mathrm{m}^{-2} \cdot \mathrm{h}^{-1}\right)$. Cabe ressaltar que a TAS não é um parâmetro de dimensionamento dos reatores UASB, porém as velocidades ascensionais são adotadas como parâmetros de verificação. De maneira teórica e a partir do cálculo hidráulico, pode-se afirmar que a TAS é um parâmetro semelhante à velocidade ascensional do líquido. Campos (1999) estudou as velocidades ascensionais em reatores UASB de maneira experimental e chegou a valores de $0,5 \mathrm{~m}^{3} \cdot \mathrm{m}^{-2} \cdot \mathrm{h}^{-1}$ a $0,7 \mathrm{~m}^{3} \cdot \mathrm{m}^{-2} \cdot \mathrm{h}^{-1}$, para vazões médias, ou $0,9 \mathrm{~m}^{3} \cdot \mathrm{m}^{-2} \cdot \mathrm{h}^{-1}$ a 1,1 $\mathrm{m}^{3} \cdot \mathrm{m}^{-2} \cdot \mathrm{h}^{-1}$ para vazões máximas. Posteriormente, a NBR 12.209:2011 passou a indicar valores inferiores a $0,7 \mathrm{~m}^{3} \cdot \mathrm{m}^{-2} \cdot \mathrm{h}^{-1}$ para vazões médias e inferiores a $1,1 \mathrm{~m}^{3} \cdot \mathrm{m}^{-2} \cdot \mathrm{h}^{-1}$ para vazões máximas. Percebe-se então que os valores teóricos de TAS apresentados na Tabela 10 se aproximam dessas referências. A elevação das velocidades ascensionais pode impactar na maior perda de sólidos no efluente (CHERNICHAR0, 2016; ALMEIDA et al., 2018; VERSIANI, 2005). Porém, a adoção de cargas hidráulicas muito reduzidas implicaria em unidades de área muito grande, perdendo a finalidade do reator UASB, que é considerado um reator compacto e de alta taxa.

De acordo com a Tabela 9 é possível verificar também, que quanto maior o TDH do sistema, menor é a taxa aplicada por área nessa unidade. Isso pode ser claramente observado, ao se comparar os valores de TAS entre as condições experimentais 01 a 05 que apresentam Profundidade de 4,0 m e entre as condições experimentais 06 a 10, que apresentam profundidade útil de $6,0 \mathrm{~m}$. 
As condições experimentais $01(\mathrm{TDH}=6,0 \mathrm{~h}$ e $\mathrm{H}=4,0 \mathrm{~m})$ e $09(\mathrm{TDH}=9,0 \mathrm{~h}$ e $\mathrm{H}=6,0 \mathrm{~m})$, dos reatores UASB alcançam os valores de TAS idênticos e de COV próximos, apresentando assim uma eficiência de tratamento similar para sistemas com condições distintas. A partir desses resultados, é possível observar que, na condição 09, apesar do aumento da profundidade, essa permitiu a adoção de uma unidade de tratamento com maior tempo de detenção hidráulica, considerando uma mesma vazão aplicada, e mesma área aplicada em comparação a uma unidade com menor profundidade, como a condição 01. Porém, cabe salientar, que os TDH para os reatores UASB são parametrizados em relação à temperatura média do mês mais frio e não devem ser alterados desrespeitando esse critério (CHERNICHAR0, 2016). Ainda, TDH muito elevados podem levar à maior formação de escuma nos compartimento de digestão (ALMEIDA et al., 2018).

Quanto maior o TDH dos Reatores UASB, menor é a COV, para uma concentração de matéria orgânica compatível com a qualidade média dos esgotos domésticos no Brasil. Valores apresentados por Chernicharo (2016); Costa (2009); Jordão e Pessoa (2017) para COV em Reatores UASB encontram-se na faixa entre 2,5 e 3,0 $\mathrm{kg} D Q 0 . \mathrm{m}^{-3} \cdot \mathrm{d}^{-1}$. Perceba que a COV sugerida por esses autores é estabelecida em relação à $\mathrm{DQO}$ e não à $\mathrm{DB} 0$. Assim, considerando-se uma relação DQ0/DB0 de esgoto da ordem de 2,0 (VON SPERLING, 2017), os valores teóricos calculados para esse parâmetro se encontram em faixas razoavelmente similares, variando de 1,4 kg DQ00.m ${ }^{3} \cdot \mathrm{d}^{-1}$ a $2,4 \mathrm{~kg} \mathrm{DQ} 00 \cdot \mathrm{m}^{-3} \cdot \mathrm{d}^{-1}$.

Ainda, segundo estudos realizados, é de suma importância que os reatores UASB apresentem um tempo de retenção superior a 4,8 horas pois essa condição evita o arraste de lodo do sistema e aumenta a residência celular dentro dessa unidade (VERSIANI, 2005; CAMPOS, 1999).

Em relação às lagoas de estabilização, alguns autores (MELO Jr. et al., 2018; PERÍGOLO, 2004) ressaltam que os resultados de dimensionamento realizados por diferentes métodos empíricos têm demonstrado grandes variações entre eles. Em geral, conforme já mencionado, no Brasil, não há parâmetros definidos em Normas Técnicas, cabendo as indicações para COV, TAS, TA0 e TDH, à literatura disponível. Espinosa et al. (2017) estudaram o comportamento de 388 lagoas em escala plena e concluem que futuras pesquisas são necessárias para um melhor entendimento da influência do projeto, da operação e dos fatores ambientais no desempenho da unidade. 
Nos sistemas de lagoas anaeróbias, ao se elevar as COV, as TAS se elevam também. Entretanto, os TDH são reduzidos. Assim, ao se analisar as condições 04 à 12 das lagoas anaeróbias, com $\mathrm{COV}$ superior à $0,2 \mathrm{KgDB} 0 . \mathrm{m}^{-3} \cdot \mathrm{d}^{-1}$, os TDH calculados não atendem aos limites mínimos encontrados nas bibliografias (VON SPERLING, 2017; JORDÃO E PESSOA, 2017). Esse deveria estar no intervalo de 3 a 6 dias.

Ao se relacionar as lagoas de polimento com as lagoas facultativas, percebe-se que as primeiras assumem a função de um sistema de pós tratamento, recebendo efluentes com cargas orgânicas reduzidas provenientes de unidades antecedentes. Assim, as lagoas de polimento apresentam TDH reduzidos se comparadas às lagoas facultativas, podendo ser até 5 vezes inferiores. Estudos realizados por Kato e Florencio (2001) mostraram que quando aumentados os TDH em lagoas de polimento não há diferença significativa de eficiência em termos de remoção de DQ0, mas apresentam melhores resultados para remoção de coliformes.

É possível verificar na Tabela 11 que para a condição 05 da lagoa facultativa, onde é adotada alta taxa de aplicação orgânica $\left(350 \mathrm{~kg}\right.$ DB0.ha- $\left.{ }^{-1} \cdot \mathrm{d}^{-1}\right)$ e baixa profundidade $(1,5 \mathrm{~m})$, é encontrado tempo de retenção muito reduzido, que não atende ao tempo mínimo proposto na literatura (VON SPERLING, 2017; JORDÃO E PESSOA, 2017). Uma situação oposta é verificada na condição 06 da lagoa facultativa, onde é adotada baixa TA0 (100 kg DB0.ha $\left.{ }^{-1} \cdot \mathrm{d}^{\mathrm{l}}\right)$ e elevada profundidade $(2,0 \mathrm{~m})$, encontrando-se tempo de retenção elevado, que ultrapassa o valor máximo proposto na literatura (VON SPERLING, 2017; JORDÃO E PESSOA, 2017).

\section{CONCLUSÃ0}

De acordo com os resultados encontrados, observou-se a partir dos cálculos teóricos, que nos decantadores primários e nos compartimentos de decantação dos reatores UASB é possível adotar áreas mais reduzidas, mantendo a eficiência de sedimentação das partículas, se implementadas unidades mais profundas. Ainda em relação aos decantadores, para se alcançar os valores de TDH máximos determinados pela NBR 12.209:2011 seria necessária a adoção de profundidades demasiadamente elevadas, que em geral não são praticadas.

Em relação às lagoas, por apresentarem critérios de dimensionamento basicamente empíricos, observou-se um descompasso entre os parâmetros estabelecidos na literatura. Para as lagoas anaeróbias, valores de COV e TAS muito elevados, levam à TDH demasiadamente reduzidos. Para as lagoas facultativas, adotando-se TA0 próxima ao limite superior e 
profundidade próxima ao limite inferior, o TDH não atinge o limite mínimo recomendado; de maneira oposta, TA0 reduzida e profundidade elevada, levam à extrapolação de TDH.

Por fim, destaca-se que o desenvolvimento do projeto de ETE não se baseia somente na aplicação de valores recomendados para parâmetros de dimensionamento em equações clássicas estabelecidas. 0 entendimento da relação entre eles é relevante para o sucesso dos cálculos.

\section{REFERÊNCIAS BIBLIOGRÁFICAS}

ABNT. NBR 12.209:2011: Elaboração de projetos hidráulico-sanitários de estações de tratamento de esgotos sanitários. ABNT. Rio de Janeiro: 2011.

ALMEIDA, P.G.S.; RIBEIR0, T.B.; SILVA, B.S.; AZEVEDO, L.S.; CHERNICHAR0, C.A.L. Contribuição para o aprimoramento de projeto, construção e operação de reatores UASB aplicados ao tratamento de esgoto sanitário - Parte 6: Qualidade do efluente. Revista DAE, v. 66, n. 214, nov/2018.

ANA. Atlas esgotos: despoluição de bacias hidrográficas. Brasília: ANA, 2017.

BECKER,F.A.; HEDGES,P.D.; SMISSION,R.P.M. The distribution of chemical constituents within the sewage settling velocity grading curve. Water Science and Technology, v. 33, n. 9, p. 143-146, 1996.

BRASIL. Ministério das Cidades. Secretaria Nacional de Saneamento Ambiental (SNSA). Sistema Nacional de Informações sobre Saneamento: Diagnóstico dos serviços de água e esgoto. Brasília: SNIS, 2019.

CAMPOS, J. R. Tratamento de Esgotos Sanitários por Processo Anaeróbio e Disposição Controlada no Solo. 1 ed. Rio de Janeiro: ABES, PROSAB, 1999.

CHAVEZ, A.; MAYA, C.; JIMENEZB. Particle size distribution to desing and operate APT process for agricultural wastewater reuse., Water Science and Technology, v. 53, n. 7, p. 43-49, 2--6.

CHERNiChaR0, C. A. L. Reatores Anaeróbios. 2 ed. Belo Horizonte: Departamento de Engenharia Sanitária e Ambiental, UFMG, (Princípios do tratamento biológico de águas residuárias); v.5. 2016.

COSTA, E. S. Proposta de Protocolo para Projeto de Engenharia de Reatores Anaeróbios de Fluxo Ascendente com Manta de Lodo (2009). Dissertação (Mestrado em Engenharia Ambiental). Universidade do Estado do Rio de Janeiro - UERJ. Rio de Janeiro.

ESPINOSA, M.F.; SPERLING, M.; VERBYLA, M.E. Performance evaluation of 388 full-scale waste stabilization ponds systems with seven different configurations. Water Science and Technology, v. 75, n. 4, p. 916-927, 2017.

FIGUEIRED0, I. C. Avaliação de desempenho do processo CEPT (Chemically Enhanced Primary Treatment) no tratamento primário de esgotos domésticos (2009). Tese (Doutorado em Engenharia Civil) Universidade Federal do Rio de Janeiro - COPPE/UFRJ. Rio de Janeiro.

GOBEL, A.; MCARDELL, C.S.; JOSS, A.; SIEGRIST, H.; GIGER, W. Fate of sulfonamides macrolides and trimethoprim in different wastewater treatment technologies. Science of the total environment, v. 372, issues 2-3, p. 367-371, jan/2007.

HELLER, L. CASTR0, J. E. Política pública e gestão de serviços de saneamento. Edição ampliada. Belo Horizonte: Editora UFMG; Rio de Janeiro: Editora Fiocruz. 2013

JORDÃO, E.P e PESSÔA, C.A. Tratamento de Esgotos Domésticos. 7. ed. Rio de Janeiro: ABES, 2017. 
Análise Comparativa de Parâmetros Hidráulicos para Dimensionamento de Tecnologias em Estações de Tratamento de Esgoto

KATO, M e FLORENCIO, L. Pós-tratamento de efluente anaeróbio em Lagoa de Polimento. In: PROSAB - Coletânea de Trabalhos Técnicos. Volume 2. 2001.

KUCZMAN, 0; GOMES, S.D.; TAVARES, M.H.F.; TORRES, D.G.B.; ALCÂNTARA, M.S. Produção específica de biogás a partir de manipueira em reator de fase única. Engenharia Agrícola, v. 31, n. 1, p. 143-149, fev. 2011.

MLEO JR., A.S.; FILHO, R.C.B.; PAPADOPOLI, G.U.; MORAIS, A.C.; HOCK, L.B.J.; CHIRINOS, G.J. Avaliação da demanda bioquímica de oxigênio (DBO) em uma lagoa facultativa. INOVAE - Journal of Engineering Architecture and Technology Innovation, v. 6, JAN-DEZ 2018.

METCALF \& EDDY. Wastewater engineering: treatment and resource recovery. 1 ed. New York: McGraw-Hill Education 2014.

PERÍG0LO, R. A. Avaliação do Emprego de Lagoas de Estabilização em Escala Piloto para Pesquisa de Tratamento de Esgoto Doméstico. Dissertação de Mestrado. Universidade de Brasília, 2004.

SANTOS, H.R.; PRADO, G.S.; VIDAL, C.M.S; MORUZZI, R.B.; CAMPOS, J.R. Aplicabilidade das técnicas de determinação de tamanho de partículas em sistemas de tratamento de água e esgoto. Engenharia Sanitária e Ambiental, v. 9, n. $4, \mathrm{dec} / 2004$.

VERSIANI, B. M. Desempenho de um Reator UASB Submetido a Diferentes Condições experimentais Tratando Esgotos Sanitários do Campus da UFRJ (2005). Dissertação (Mestrado em Engenharia Civil) Universidade Federal do Rio de Janeiro - COPPE/UFRJ. Rio de Janeiro.

VON SPERLING, M. Introdução à qualidade das águas e ao tratamento de esgotos. 4. ed. Belo Horizonte: Departamento de Engenharia Sanitária e Ambiental, UFMG (Princípios do tratamento biológico de águas residuárias); v.l. 2014.

VON SPERLING, M. Lagoas de Estabilização. 3. ed. Belo Horizonte: Departamento de Engenharia Sanitária e Ambiental, UFMG, v.3. 196 p. 2017.

VON SPERLING, M. Princípios básicos do tratamento de esgotos. 2. ed. Belo Horizonte: Departamento de Engenharia Sanitária e Ambiental, UFMG (Princípios do tratamento biológico de águas residuárias); v.2. 211 p. 2016. 